\title{
Index
}

Afghanistan 58-9, 64, 68-9, 86-8, 92-5, 162

Africa 28, 150, 174

Ait Ahmed, Hocine 143

Akef, Mohamed 127

Al Aqqad, Mahmoud Abbas 17

Algerian Afghanis 94-5, 120

Algerian Army (ALN) 1-3, 11, 13, 20-3, 86, 97, 110, 128, 135, 139-41, 143, 167-8, 170, $174,186,189$

Al Jazeera 171

Al Qaeda 56, 64, 148

AMOCO 164

Andarko 164

Arab League 84, 174

Arab world 3, 6-9, 12, 14-15, 34, $39,41,52,64,83,85-7$, 92-3, 95, 101-2, 113, 120-1, $123-7,144-7,161,166,168$, 184,191

Arco 158, 164

authoritarianism 7, 9-10, 13-15, $39,67,128,132-3,139,142$, $146,166,175,178$ n.5, 182, 191

Baker, James 101, 112

Belkheir, Larbi 110

Ben Bella, Ahmed 97

Bencherif, Osman 95

Benflis, Ali 172

Benhadj, Ali 114, 136, 177

Bennabi, Malek 18, 187

Benjedid, Chadli 1, 2, 19, 20-2, 76, 82-6, 91, 97, 102-4, 106-9, $116,160,185-6,189$

Berber minority 142-3 political agitation 89

Bin Ali, Zine El Abidine 5

Bin Laden, Osama 60
Boudiaf, Mohamed 145

Bouteflika, Abdelaziz 3, 154, 174, 176-8

Brahimi, Lakhdar 146

Brazil 30-1

British Petroleum (BP) 55, 158, 164

Burma 49

Bush, George H. 84, 101-2, 104, $111-12,161,163$

Bush, George W. 126, 147n.1, 174

Canada 79, 133

capitalism 45, 67 crony capitalism 159, 169, 175-7

Carter, Jimmy 61

Central Intelligence Agency (CIA) 58-9

Chavez, Hugo 126

China 49, 55, 58, 67

Chirac, Jacques 149, 161, 174

civil society $2,10-13,34,50,63$, $135,151,153,164,166,171$

civil war 3-4, 40, 120, 144, 152, $158,168,170-1,175,178$, 184,190

clash of civilisations 65,166

Clinton, Bill 163

Cold War 6, 7, 27, 48, 55, 58, 60-4, $70,82,87,101-4,120-1,150$, 162,185

Colombia 65

Constitution of Algeria 2, 67, 76-7, $91,104,106,174$

constitutional coup 3, 21, 112, $140,161,190$

coup d'état $3,6,12,156$

corruption $67,90,92,118,141$, 174-7

democracy promotion 48, 61-3, 65, $70,100,105 a v 112 n t 61,9165847792778$ 
democratisation 2-4, 6-7, 9-13, 17, $19,22-4,27-8,31-42,48-9$, 54, 62-3, 68-9, 80-1, 91, 96, 105-8, 117-21, 123-8, 137, $139,144,148-9,153-5,157$, $159,161,163,165,170-1$, $175,181-7,191$

Desert Storm 95, 100

direct active policies 56, 62, 105, $112,115,121$

Dumas, Roland 160

Eastern Europe 12, 27-8, 62-3 economic crisis $20,57,75,89-92$, $97,106-7,117,120,155-8$, 184,188 external debt 57, 79, 155, 176 inflation $77-8$

oil counter-boom 184

unemployment 78, 151, 177 economic position $29,45-6,80$ economic reforms $1,6,45,67$, 70-1, 75-82, 91, 117-18, 121, $151-2,159,163,175,185$ liberalisation 57, 77-8, 81, 105-6, $117,159,165,175-7$

privatisation $57,117,157,159$, 175

Ecuador 66

Egypt 5, 55, 111, 124, 126-7, 140, 147 n.2, 188

end of history 67,82

European Union 39, 43, 49, 61, $107-8,154,157,174,191$

association agreement 165-6, 177 democracy promotion $28,62,63$, $126,149,165-6$

Euro-Med Partnership and ENP $54,151,165,169$

external environment $7-8,25,33$, $36-7,40,45,49,52,73,180$, $185-6$

external shocks 56-7, 89-90, 92, 183

façade democracy $169,174-5$

family code 142-3

foreign investments $58,77,79,118$, 163

France 39, 71, 78-9, 81, 100, 106-7, $121,143,145,149,154,161$, 166

elections in Algeria 108

Gulf War 86, 99, 109

financial assistance 97, 156, 160, $163-4,186$ military assistance 102-3, 167, 170

relations with Algeria 80, 102, $105,110-11,156,160$

Front de Libération National (FLN) 2, 10, 18-20, 94, 103, 109, $132,142,171-2$

Front des Forces Socialistes (FFS) 173

Front Islamique de Salut (FIS) 2, 7, 11-13, 21-3, 71, 81, 83, $85,90,93-5,110,113-15$, $120,123,128,132,138,147$, 151-4, 169-73, 186-9

elections $3,70,86,95,112,124$, $129,133-5,191$

Gulf crisis 96-100

leadership 18, 21, 84, 90, 114, $136,145,160-3,177$

peace platform 164 policies 21-2, 78-80, 91, 104-9, $111,118,121,139-44$ violence 146, 155

geopolitical environment $38,47-8$, $52-6,82$

Ghazali, Mohamed 142

Ghozali, Sid Ahmed 20

Giscard d'Estaing, Valery 140

globalisation 27, 44-5, 54, 181

Gorbachev, Mikhail 27, 82, 101, 150

Great Britain 59, 100, 102, 164 see also United Kingdom

Groupes Islamiques Armés (GIA) 144

Guatemala 66

Gulf monarchies 96, 187

Gulf states $63,87,96,113,115$, 142,168

Gulf War 60, 69, 83-6, 95, 98-100, $105,109,111,120,186,189$

Hachani, Abdelkader 95, 145, 160

Hamas 124, 126

Hamilton, Lee 112

Hamrouche, Mouloud 20-2, 76, $80-1,107$

Hassan II, King 5

Hidouci, Ghazi 20-2, 81

Hussein, Saddam 16, 84, 95-8, 108, 111,188

hydrocarbons $37,39,52,73-4$, 77-80, 89-90, 119, 143, 157, 164 
India 55

international factors 27-9, 31-2, 35, $37,39,181,185-6$

international variables 9,25 , 27-9, 31-3, 35, 38, 41, 47, 72, $180-1,184$

see also external environment

International Monetary Fund (IMF) 43, 66, 76-7, 116-19, 156, $165,176-7$

structural adjustments programmes 57-8, 66, 116, 155

Iran 58, 63-4, 69, 86-7, 113, 125, $140,142,168$

revolution $54,63,86-8,113$, 120,187

Iraq 16, 60, 63, 69-70, 84, 95-9, $108,115,143,155$

Islamism 18, 54, 58-9, 64-5, 86-7, 92-3, 98, 108, 112-3, 123-4, $129,131,133,135,144,153$, 162,166

Islamist movements $15-6,54,64$, $93,112-13,115,121,128$, $130-2,134,161$

Islamo-technocrats 94-5, 118

Israel 96, 127, 152

peace process 105

Italy 79, 131, 156, 158

\section{Japan 79}

jihad 58-9, 87, 92, 98, 142

Jordan 111, 127

Jospin, Lionel 140

Juppé, Alain 140, 161, 164

Kabilye 142-3, 171, 173

Khediri, El Hadi 135

Khomeini, Ruhollah 16, 63, 87, 93, 142

Kuwait 60, 69, 83-4, 96-7

Lamari, Mohamed 110, 139

Lamari, Smain 175

larger trends $33,56-7,66,71,183$

Latin America 28, 30-1, 62, 150

liberalism 43, 125-6

Libya 5, 55

Madani, Abassi 91, 93, 99, 104, 114, $136,142,144$

Maghreb 53, 56, 111, 149, 162

Mediene, Mohamed 175

Mediterranean 49, 152, 163, 165

geopolitics 52-5

Messaoudi, Khalida 139, 164
Middle East and North Africa

(MENA) 5, 7-8, 50-1, 55, 60, $154,184,191$

Mitterrand, François 99, 103, 106-7, 109-10, 112, 121, 139, 160,163

Morocco 5, 56, 127

Mouvement National pour la Réforme (MNR) 172

Mouvement de la Societé pour la Paix (MSP) 172

multinational companies 34, 115

multinationals 44, 48, 50, 55, 79-80, 105, 115-16, 118, 121, 157,177

see also transnational companies

Muslim Brotherhood 126-7, 130

national unity $10-11$

neo-liberalism 45, 151

New Economic Order 103

New World Order 70, 101

Nezzar, Khaled 21-3, 98, 110, 135, 139,189

Nicaragua 61

Nigeria 66

North Atlantic Treaty Organisation (NATO) 152, 178

North Korea 49

October 1988 riots $23,89-90$

oil prices 52, 74-5, 91-2, 117, 155

Organisation of Oil Exporting Countries (OPEC) 46

Pakistan 59, 93

Palestine 115, 126, 143

path-dependency 9, 19, 23, 41, 56

Peace and National Reconciliation 178

pivotal state $48-9,55$

political culture 9, 14-15, 18, 23, 42

Portugal 28

Pouvoir, le 110, 145

power-sharing 22, 189

preconditions of democracy 9, 11, 13

Qadahafi, Muammar 5

Qatar 168-9

Qutb, Sayyid 16-17

Rassemblement National

Démocratique (RND) 172

Rassemblement pour la Culture et la Démocratie (RCD) 135,

Francesco Cavatorta - 9781847792778 
139

Reagan, Ronald 61, 116-17

Redjam, Abderrazak 145

rentier state $37,49-52,57,73,75$, 91

rentierism $37,49,51-2,153$

Rice, Condoleeza 126, 147n.3

Rocard, Michel 107, 160

Rondot, Philippe 160

Sadi, Said 143

Said, Mohamed 145

Saint Egidio peace talks 153, 164

Sarkozy, Nicolas 149

Saudi Arabia 6, 60, 63-4, 84, 96, 113-15, 143

September 11th 14, 61-2, 65, 152

SNAM 158

socialism 66-8, 70, 82, 86, 103-4, $113,159,185$

soft-liners 1

SONATRACH 77, 79, 81

Soroush, Abdolkarim 17

Southern Europe 28, 53-4, 79, 164

Soviet Union 3, 27, 39, 59, 60, 67, $70,82,87,100-3,162,169$

Spain 28, 156

Sudan 168

Syria 6

\section{Thatcher, Margaret 117}

Third Worldism 103, 149

Total 158

Touati, Mohamed 110

transition 3, 6-8, 10-12, 46-7, 53, $55,57,61,63,69,71-4,76$, 78-81, 83-4, 86, 95-6, 98, $101,106-9,113,116-17$,
120-1, 123-4, 126, 128, 132, $135-6,138,150,189$

failure 4, 68, 119, 123, 134, 148

literature 5, 9, 18-41, 180-7

pact 24,72

transitology 4, 5, 33, 124, 181

transnational companies 65,71

Tunisia 5, 24, 56, 127

Turkey 110-11, 182

Tutwiler, Margaret 112, 161

United Kingdom 72n.1, 79, 114, 117,167

United Nations 60

United States 39, 43, 49, 53-5, 58-9, 64-5, 70-1, 79-80, 82, $87,97,100-6,111-12,121$, $124,126,140,143,149,156$, $162-4,167$

Algerian elections 86, 161

democracy promotion $6,61-2$, $117,154,163$

Iraq 60, 69, 83-4, 96-7, 106, 155 war on terror $61,149,152,154$, $169,178,191$

urbanisation 10-12, 58

Vedrine, Hubert 106

Venezuela 68, 126

war on terror $53-5,72,103,121$, $149,152,155,166,178,191$

World Bank (WB) 43, 66, 76, 116, 156,165

World Systems Theory 31-3

Zeroual, Liamine 159, 163, 169-72 